\title{
A Comprehensive and Numerical Modeling of Reactive Polymer Flow in Porous Media
}

\author{
Reza Cheraghi Kootiani ${ }^{1}$, Ariffin Bin Samsuri ${ }^{2}$ \\ ${ }^{1,2}$ (Department Of Petroleum Engineering, Faculty of Petroleum and Renewable Energy Engineering, \\ University Technology Malaysia.
}

\begin{abstract}
In the present paper, an overall model for the study of a reactive polymer flow across a heterogeneous porous medium is proposed. A moment representation is used to simulate the polymer transport and reaction processes in a two dimensional porous medium. The 2D, multiphase polymer flow model is based on a mass-transport equation for multicomponent species and is coupled with kinetic models of the gelation process using an operator splitting scheme. This model leads to using method of line technique to solve the set of equations.
\end{abstract}

Keywords: - polymer transport, crosslinking, molecular weight distribution, moments, porous media

\section{INTRODUCTION}

The flow of reactive polymers through porous media is of interest to engineers because of practical applications such as hydraulic fracturing, materials impregnation, and filtration processes [1]. For example, reactive resins are used during the production of certain types of composite materials. How the liquid phase moves through the matrix is essential to these applications. The dynamics of reactive polymer flow in porous media is difficult to model, especially for heterogeneous materials. The traditional approach to modeling aqueous solutions of reactive polymers in porous media has been to consider the polymer as a single-component species $[2,3]$. However, most polymers are polydisperse, and the transport properties of lower and higher molecular weight species are known to be different. In order to effectively model polymer transport and gelation in porous media, it is important to address effects associated with polydispersity and evolution of the molecular weight distribution (MWD) due to crosslinking reactions. An approach for modeling polymer reactions is to use the polymer MWD, which can be described by moments of that distribution. In this work, we describe a new method for modeling reactive polymer flow in heterogeneous porous materials. We use a moment representation of the log-normal polymer MWD to model polymer as a multi-component species. The 2D, two-phase polymer flow model is based on a mass-transport equation for multi-component species. It is coupled with kinetic models of the crosslinking process by using an operator splitting scheme.

\subsection{MODELING OF FLOW IN POROUS MEDIA}

\section{MODELING}

The modeling of flow in porous media is required in a number of chemical engineering processes. A continuum representation of porous media is typically used in which the conservation equations are combined with the necessary constitutive equations for transport. The flow of two fluid phases (wetting and non-wetting) can be described by the continuity equation and Darcy's equation for each fluid phase as:

$$
\begin{aligned}
& \frac{\partial\left(\emptyset \mathrm{p}_{\alpha} \mathrm{s}_{\alpha}\right)}{\partial \mathrm{t}}=-\nabla \cdot\left(p_{\alpha} u_{\alpha}\right) \\
& u_{\alpha}=-\frac{k_{r \alpha} K}{\mu_{\alpha}}\left(\nabla P_{\alpha}-p_{\alpha} g\right)
\end{aligned}
$$

Where the subscript $\alpha$ indicates the various phases in the system. Here we assume $\alpha$ takes the values $\mathrm{w}$ and nw (wetting and non-wetting). The relative permeabilities are strong functions of pore structure and saturation, and can also depend on saturation history, capillary number, and viscosity ratio, the saturations of the fluid phases are related by $\sum S_{\alpha}=1$, and the pressures of different fluid phases are related by the capillary pressure:

$$
\text { 1. } \quad P_{c}\left(S_{\alpha}\right)=P_{n w}\left(S_{\alpha}\right)-P_{w}\left(S_{\alpha}\right)
$$




\subsection{MODELING OF REACTIVE TRANSPORT IN POROUS MEDIA}

The modeling of reactive transport in porous media is challenging because of the dynamic coupling that can occur between flow, reaction, and pore structure. The general convection-dispersion-reaction equations for multi-component systems can be written for each phase as:

$$
\frac{\partial\left(\phi S C_{i}\right)}{\partial t}=-\nabla \cdot\left(u C_{i}\right)+\nabla \cdot\left(\varnothing D S \Delta C_{i}\right)+\emptyset S R_{i}
$$

Where the subscript i denotes a solute species (or in this application, a discrete range of polymer molecular weights). Normally, the partial differential equations for transport without the reaction term are solved by Eulerian methods, for example finite difference or finite element methods; a grid or mesh is constructed representing the spatial domain, and then concentrations are updated by stepping through time. An accurate solution of Eq. (3) requires that the reaction and transport terms be coupled at some level, because they affect each other. Several methods have been proposed to solve the coupled set of equations. One of them can be written as:

$$
\begin{aligned}
& \frac{C_{i}^{i}-C_{i}^{n}}{\Delta t}=L\left(C_{i}\right)^{n} \\
& \frac{c_{i}^{n+1}-C_{i}^{\prime}}{\Delta t}=R_{i}^{n+1}
\end{aligned}
$$

Where $\mathrm{L}$ is the spatial operator. The sequential iteration approach (SIA), where iteration is performed between the reaction and transport calculations, has been suggested to reduce the operator splitting errors; this approach can be numerically unstable.

\subsection{METHOD OF MOMENTS}

In most polymeric fluids, polymer chain length is a distribution, which we denote $\left[P_{i}\right]$ by and $(\mathrm{i}=1, \infty)$ the molar concentration of species of length i [4]. The polymer chain length distribution can be described by moments $\left(\mu_{j}\right)$ :

$\mu_{j}=\sum_{i=1}^{\infty} i^{j}\left[P_{i}\right]$

We are interested in understanding how polymer transport in porous media is affected by in-situ crosslinking reactions. Conceptually we address this problem by solving a system of equations given by Eq. (3), for $\mathrm{i}=(1, \mathrm{~N})$ where $\mathrm{N}$ is the total number of solute species including cross-linker, all discrete ranges of polymer weights, and any other components that may be present in the flow. In this formulation, we make the following assumptions. First, the polymer is a dilute, aqueous-phase solution. Second, the MWD of polymer is a lognormal distribution. Third, we assume that the crosslinking reaction proceeds by a stepwise polymerization mechanism.

\subsection{GOVERNING EQUATIONS}

Assuming that all the chemical species are transported by the aqueous phase (wetting phase) only, the mass conservation equations can be written as:

$$
\frac{\partial\left(\emptyset p S_{W} C_{i}\right)}{\partial t}+\frac{\partial\left\lfloor f p_{r}(1-\emptyset) C_{i r}\right\rfloor}{\partial t}=\nabla \cdot\left(\emptyset D p S_{w} \nabla C_{i}\right)-\nabla\left(p C_{i} u\right)+\emptyset p S_{w} R_{i}
$$

Where $C_{i}$ is mass concentration of component i $(\mathrm{i}=1,2, \ldots, \mathrm{N})$, and $C_{i r}$ is the mass concentration of component $\mathrm{i}$ adsorbed on porous medium surface. The term $\mathrm{D}$ describes either diffusion or dispersion; for most convective porous medium flows, hydrodynamic dispersion is dominant, which means D should be defined as a tensor rather than a scalar to account for anisotropy. We retain the scalar form here for simplicity, noting that it is straightforward to incorporate the tensor form in the numerical equations. The left-hand side of Eq. (7) describes the time rate of change of solute and adsorbed species, respectively. The three terms on the right-hand side of the equation describe diffusion/dispersion, convection and reaction, respectively.

\subsection{GELATION REACTION KINETICS}

A simple polymer and cross linker gelation model is used for the kinetics. $P_{n}$ is polymer, and $\mathrm{X}$ was considered as cross linker [5]: 


$$
\begin{aligned}
& \frac{d\lfloor X\rfloor}{d \tau}=-\sum_{K-1}^{\infty}\left[P_{k}\right], \\
& \frac{d\left\lfloor P_{i}\right\rfloor}{d \tau}=-2\left[P_{i}\right]+\frac{2}{\sum_{K-1}^{\infty} P_{I}} \sum_{K-1}^{i-1}\left(\left[P_{i-k}\right]\left[P_{k}\right]\right)
\end{aligned}
$$

Since the focus of the current work is modeling the dynamics of flow and reaction we have used generalized auxiliary equations, which can be changed for specific situations as information is available.

\subsection{NUMERICAL SOLUTION}

To have a numerical solution of these equations, we suppose the linear reactions and then we find $\mathrm{k}$ (the reaction coefficient) with a try and error method. The numerical model has been built within MATLAB, so we can use the method of line for this reason. We convert the PDEs to many ODEs whit the loops of this program. The accuracy of these equations is $e^{-10}$, and the method of Rung- cutta $4^{\text {th }}$ order has been used.

Since the transport and reaction steps are solved explicitly, parameters must be updated before beginning a new time step. The most difficult issue to contend with is determining how in-situ crosslinking affects the morphology of the porous medium, specifically for cases where gel formation occurs because the formation of gel has a dramatic impact on hydraulic conductivity.

\section{RESULTS AND DISCUSSIONS}

To effectively model reactive polymer flow in porous media, it is important to understand the sensitivity of various parameters in the model.

\subsection{FORM OF THE MWD}

One of the most important limitations of this modeling technique is how well a given mathematical distribution can represent a true polymer weight distribution. This issue is especially significant if mixing of different compositions occurs so as to create distributions with anomalies such as multiple peaks. Four polymer MWDs frequently encountered in practice are Wesslau log-normal, Lansing log-normal, Poisson, and mostprobable Distributions [6]. In our work, we use a log-normal MWD, which is the empirical form of the Wesslau distribution. In our model, we assume that the polymer MWD remains $\log _{-}$normal during the transport processes. This restriction imposes limitations on the amount of mixing that can occur from different regions in the porous media because the mixed distributions may not be log- normal.

As an extreme example, consider two distributions: $M_{w 1}=1.63 \times 10^{6}, I_{P 1}=1.43$ and $M_{w 2}=1.63 \times 10^{7} \quad, I_{P 2}=2.43$ shown in "Fig1.", which are mixed in volume fractions $f_{1}=0.2, f_{2}=0.8$. The mixed distribution is shown in "Fig2.". For comparison, the first three moments of the true mixed distribution were calculated and used in the log- normal function, which is also shown in "Fig2."Clearly the mixed distribution cannot be described using a log-normal equation, and if a practical case exhibits this type of mixing, additional considerations will have to be made in the modeling approach.

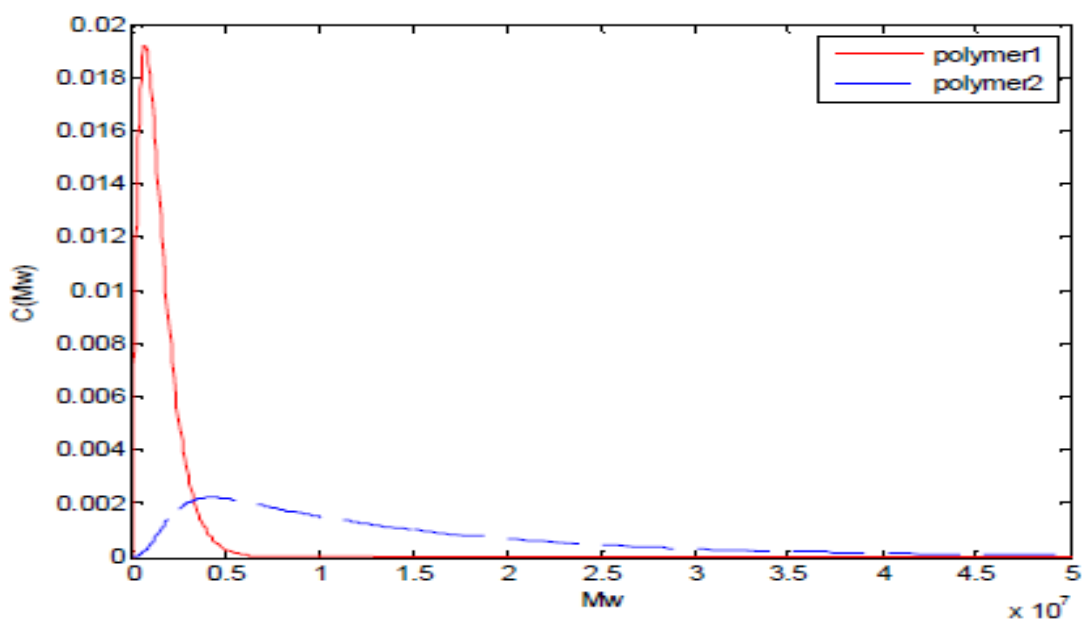

Figure1. Two log- normal distributions. 


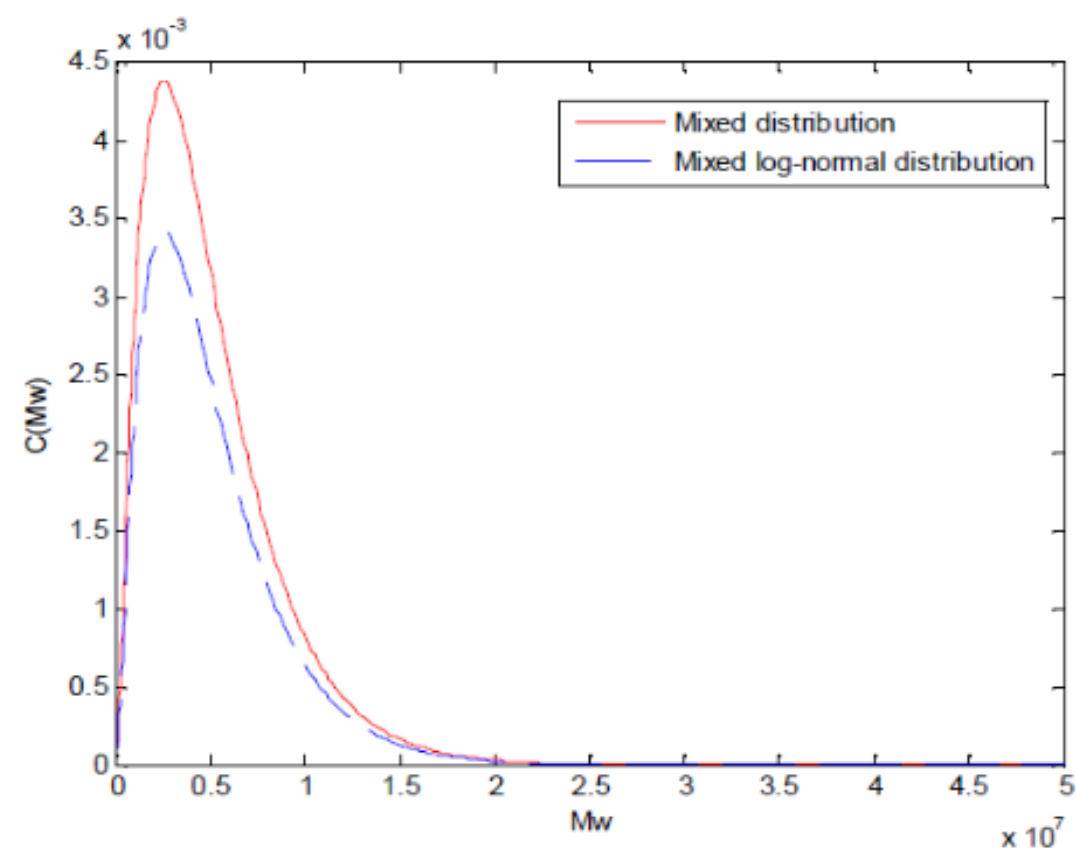

Figure2. Mixed distribution of the two log- normal distributions.

\subsection{MOBILE POLYMER TRANSPORT}

During the species transport step, only the mobile fraction of the MWD is allowed to flow, while the highest-molecular weight species are considered to be part of the gel phase. Meanwhile, the mass of immobile phase polymer should remain constant during the transport calculations of a time-step. However, the use of the compromised distribution usually causes an apparent increase in the mass of immobile polymer. "Fig3." shows an example for a representative grid block undergoing transport. It contains the pre-transport distribution, the post-transport distribution, and the log- normal distribution calculated directly from the discretized distribution. In this example, the apparent change in the mass of immobile phase due to this approximation was $67.7 \%$.

We have investigated a number of techniques for minimizing this conversion error. The approach that appears to be most effective is to calculate new moments from the discretized distribution as initial values, and then to perform an optimization procedure to search for an optimal log- normal distribution to best approximate the new distribution after transport. This optimization minimizes the least-squares error in the difference between true post-transport concentrations and the concentrations found from the log-normal equation. "Fig4." shows the results of this optimization.

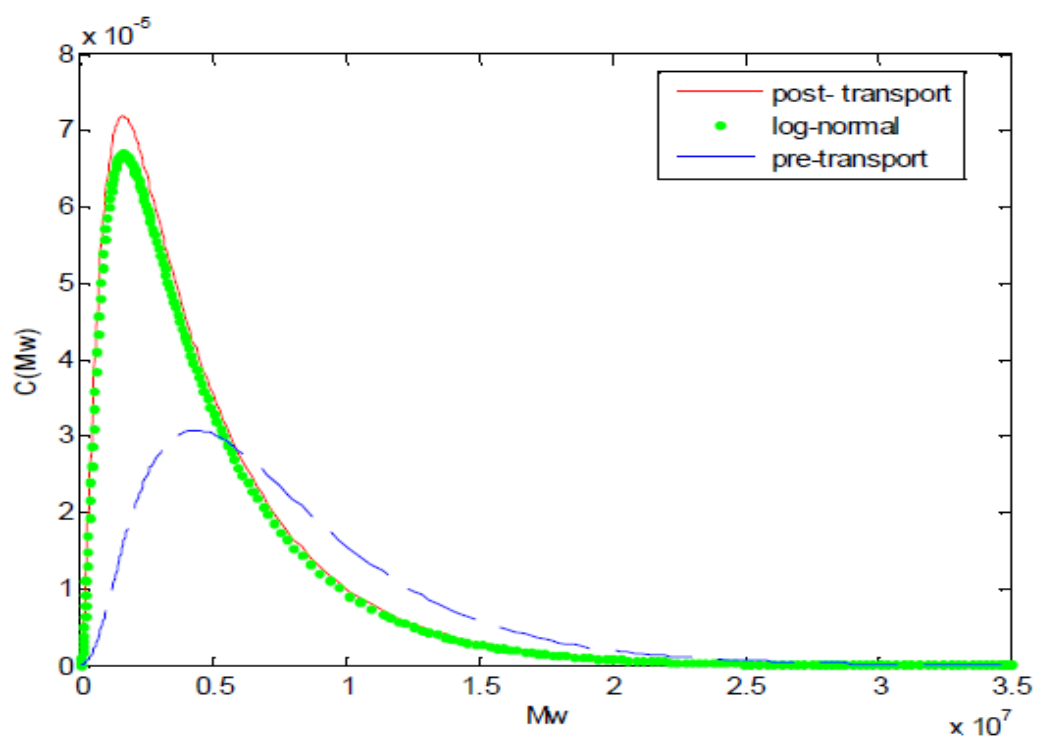

Figure3. The concentration distributions and the distribution using moments of the post- transport curve. 


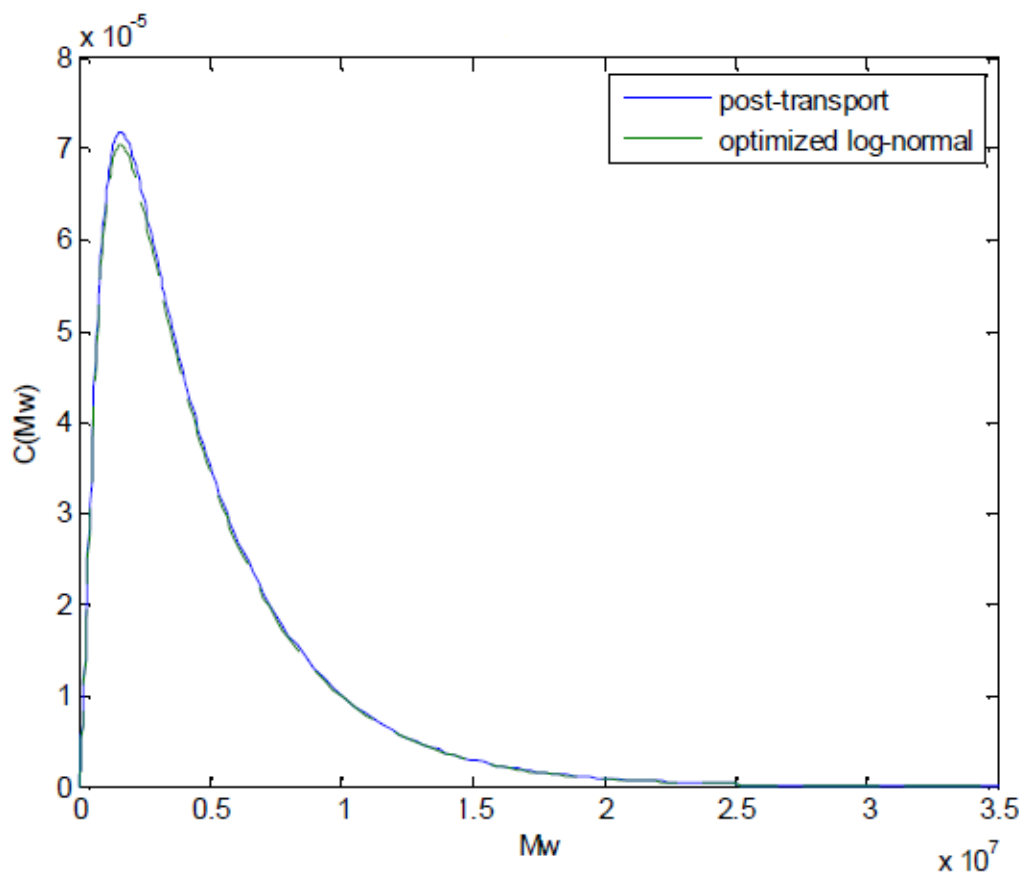

Figure4. The results of the optimization of the concentrations found from the log-normal equation.

\subsection{TIME-STEP SIZE}

Time-step size plays a unique role in this model because of differing effects in the transport versus reaction parts of the algorithm. If the time-step is large the normal truncation errors associated with the finite difference method become significant. If it is small, repeated discretization are required, which causes cumulative error in the distribution. The polymer concentration distributions in grid-blocks $(10,10)$ and $(10,30)$ at different times are shown in "Fig5." and "Fig6.", respectively. Grid-block $(10,30)$ is located in a highpermeability region, while grid-block $(10,10)$ is in a low permeability region.

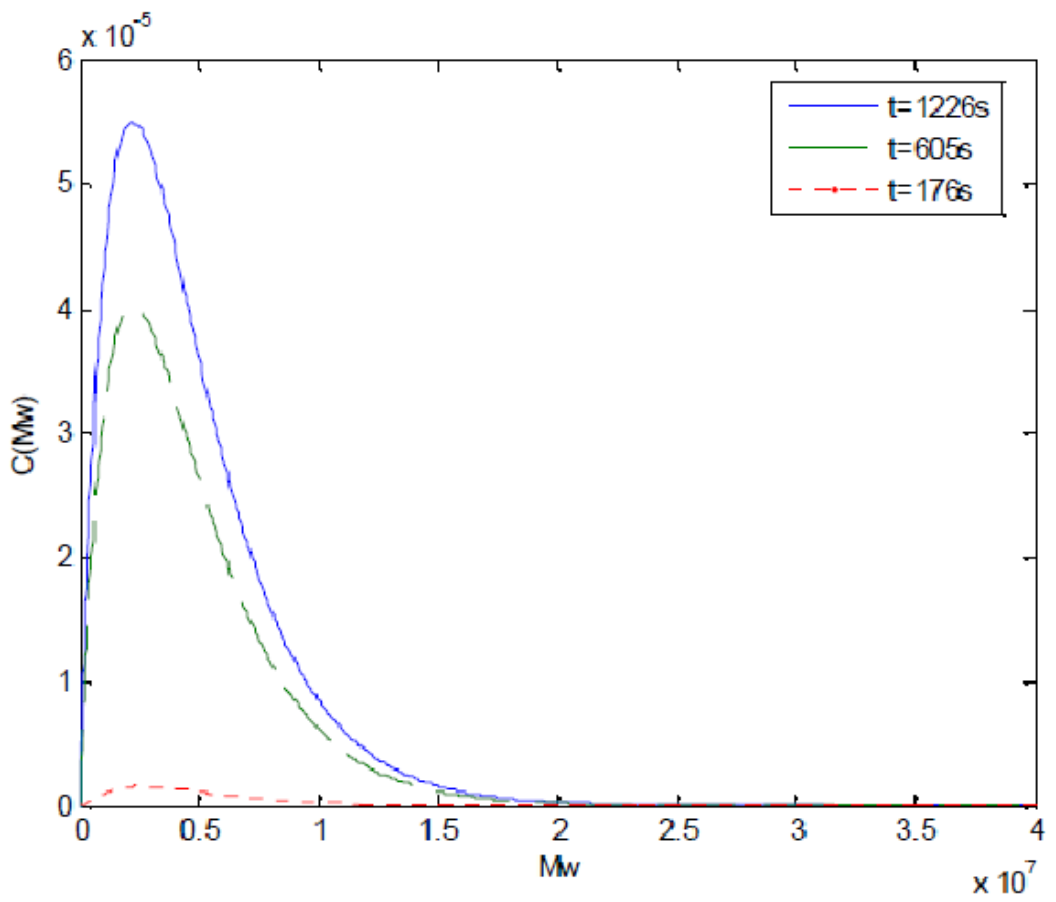

Figure5. Concentration distribution of polymer components. 


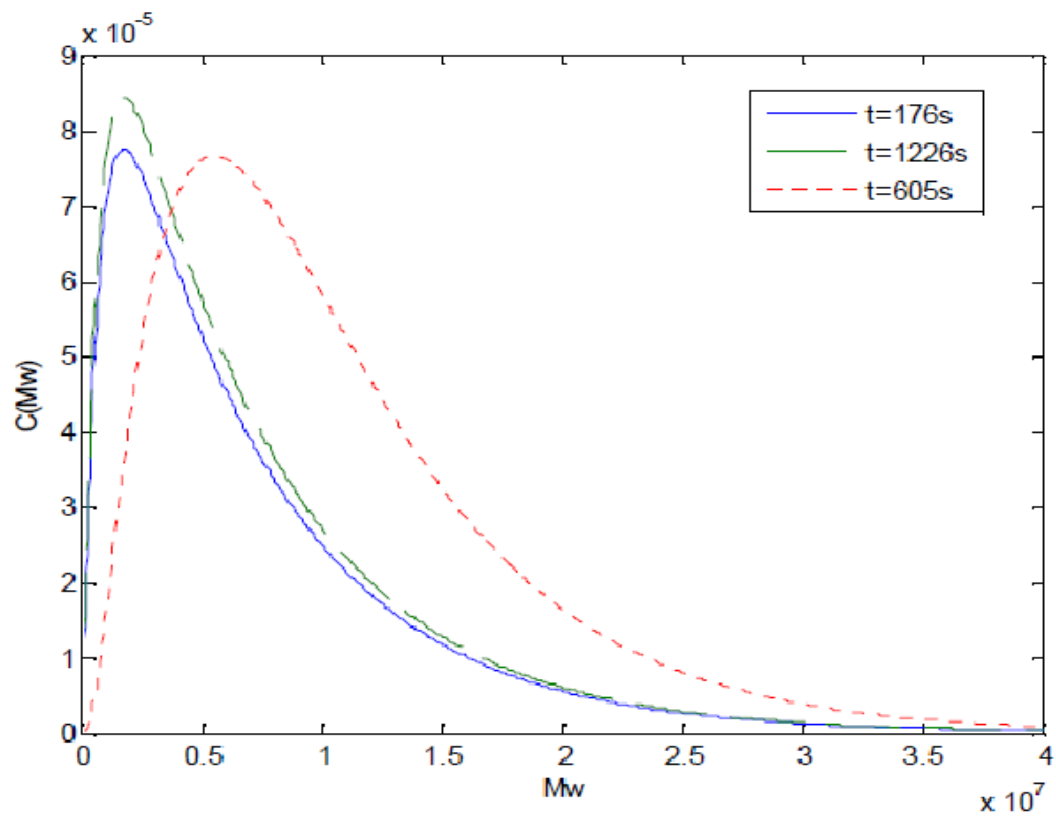

Figure6. Concentration distribution of polymer components.

By comparing "Fig5." and "Fig6.", it is noted that polymer in the high-permeability region has a higher concentration and is more polydisperse at early times. This is a transient effect. At long times, the polymer MWD in the lower-permeability region becomes much broader because of the longer residence times for fluids flowing here.

\section{CONCLUSION}

In this work, a mathematical model for two-phase flow of aqueous, reactive polymer solutions was developed. Polydispersity effects have been incorporated by using three leading moments to represent the polymer MWD. From a numerical standpoint, this is significant because only a few finite moments are needed to describe polymer transport and reaction (rather than a very large number of polymer species). Yet, order-of magnitude changes in molecular weight are accounted for accurately. The mass-transport equations for multicomponent species coupled with kinetic models of the gelation process are solved using an operator splitting scheme, in which the grid-block conditions are updated according to both flux and reaction in that order. Within each gridblock, the kinetic model is that of a batch-reactor. The polymer MWD is chosen to be log- normal and is forced to remain log- normal during the transport and reaction processes.

\section{ACKNOWLEDGEMENTS}

I would like to appreciate Universiti Teknologi Malaysia for their continual support during the course of this paper. Special thanks go to my supervisor Prof. Dr. Ariffin Bin Samsuri for his support in the publication of this paper.

\section{REFERENCES}

[1] De, D., Hrymak, A. N. \& Pelton, R. H., "A network of zones model for reactive polymer enhanced miscible displacement in a porous cylinder," Chem.Engg. Sci., 53, 3545 (1998).

[2] Brown, W. D. \& Sorbie, K. S. "Dispersion and polydispersity effects in the transport of Xanthan in porous media," Macromol., 22, 2835 (1989).

[3] Bravo, M. C., Araujo, M. and Lago, M. E., "Pore network modeling of two-phase flow in a liquid-gas system," Physica A, 375, 1 (2007).

[4] Crowley, T. J. \& Choi, K. Y. "Calculation of molecular weight distribution from molecular weight moments in free radical polymerization," Indust. Engg. Chem. Res.,36, 1419 (1989).

[5] Tang, E., Tian, B., Fu, C., "Apparent kinetic study of latex interpenetrating polymer networks Poly(ethyl acrylate)/Poly(styrene) and Poly(styrene)/Poly(ethyl acrylate) by two stage emulsion polymerization," Colloid. Surf. A: Physicochemical, 333, 7 (2009).

[6] Zabisky, R. C. M., Chan, W.-M., Gloor, P. E. \& Hamielec, A. E., "A kinetics model for olefin polymerization in high-pressure tubular reactors: a review and update," Polymer, 33, 2243 (1992). 